\title{
PREVALENCE OF BACKWARD STOCHASTIC DIFFERENTIAL EQUATIONS WITH UNIQUE SOLUTION
}

\author{
K. BAHLALI, B. MEZERDI, AND Y. OUKNINE \\ Received 20 June 2003 and in revised form 15 February 2004
}

We prove that in the sense of Baire category, almost all backward stochastic differential equations (BSDEs) with bounded and continuous coefficient have the properties of existence and uniqueness of solutions as well as the continuous dependence of solutions on the coefficient and the $L^{2}$-convergence of their associated successive approximations.

\section{Introduction}

Let $\left(W_{t}\right)_{0 \leq t \leq 1}$ be an $r$-dimensional Wiener process defined on a probability space $(\Omega, \mathscr{F}$, $P$ ) and let $\left(\mathscr{F}_{t}\right)_{0 \leq t \leq 1}$ denote the natural filtration of $\left(W_{t}\right)$ such that $\mathscr{F}_{0}$ contains all $P$-null sets of $\mathscr{F}_{\text {. Let }} \xi$ be an $\mathscr{F}_{1}$-measurable $d$-dimensional square-integrable random variable. Let $f$ be an $\mathbb{R}^{d}$-valued process defined on $\mathbb{R}_{+} \times \Omega \times \mathbb{R}^{d} \times \mathbb{R}^{d \times r}$ with values in $\mathbb{R}^{d}$ such that for all $(y, z) \in \mathbb{R}^{d} \times \mathbb{R}^{d \times r}$, the map $(t, \omega) \rightarrow f(t, \omega, y, z)$ is $\mathscr{F}_{t}$-progressively measurable. We consider the following backward stochastic differential equation (BSDE):

$\left(E^{f, \xi}\right)$

$$
Y_{t}=\xi+\int_{t}^{1} f\left(s, Y_{s}, Z_{s}\right) d s-\int_{t}^{1} Z_{s} d W_{s} \quad(0 \leq t \leq 1)
$$

Equation $\left(E^{f, \xi}\right)$ is closely connected to stochastic optimal control (via the adjoint process in the formulation of the Pontryagin maximum principle [5]) and to certain nonlinear partial differential equations (via nonlinear Feynmann-Kac formula [19, 20]); it is also used in mathematical finance. The BSDEs are also studied for their mathematical interest since nice problems still remain open.

Linear BSDEs were introduced by Bismut [5]. Pardoux and Peng [18] were the first to consider general nonlinear BSDEs in the above form. They have proved that when the coefficient $f$ is globally Lipschitz, then the $\operatorname{BSDE}\left(E^{f, \xi}\right)$ has a unique adapted and square-integrable solution. Moreover, the solution can be constructed by a successive approximations procedure, see also [8]. Since the paper [18], several works have attempted to weaken the Lipschitz condition on the coefficient $f$, see, for example, Mao 
[15], Hamadène [10], Lepeltier and San Martin [14], Dermoune et al. [7], and Kobylanski [12] and the references therein. When the coefficient $f$ is merely continuous, the questions of existence (and sometimes the uniqueness) of solutions have been partially solved for one-dimensional equations, either by comparison techniques or by using a classical transformation which removes the drift, see, for example, [7, 12, 14]. Stronger conditions are required to obtain the uniqueness. Note that the techniques used in dimension one do not work in the multidimensional case. Moreover, with the above-quoted techniques, there is no information about the convergence of the Picard successive approximation and, probably, this approximation does not converge in these situations.

In the multidimensional case, the questions of existence and uniqueness of solutions still remain largely open. Up to our knowledge, except for the papers [2, 3, 15, 20], no results are known about when the coefficient is nonuniformly Lipschitz in the two variables $(y, z)$. Moreover, in $[15,20]$, the assumptions imposed on the coefficient are global.

Our approach is quite topological but it allows the derivation of some precise examples. More precisely, we consider the set of multidimensional BSDEs, with bounded and continuous coefficients. We are then concerned with the prevalence, in the sense of Baire categories, of BSDEs which have the properties of existence and uniqueness as well as the stability of solutions and the $L^{2}$-convergence of their associated successive approximations.

Prevalence questions were studied in many areas of mathematics (see, e.g., $[1,4,6,9$, $11,13,16,21,22,23])$ and seem to take their origin from an earlier paper of Orlicz [16], where it is shown that "most" ordinary differential equations with continuous coefficient have unique solutions. In the theory of stochastic differential equations (SDE), the first result in this direction is due to Skorokhod [23], where the author has used it also to study the dependence of weak solutions on a parameter. The method developed in [23] cannot be extended to BSDEs, since it needs the notion of solutions in the sense of law and unfortunately this notion is actually not clear in BSDE's theory.

In this paper, we give an analytic approach. We consider the space of bounded $\mathscr{F}_{t^{-}}$ progressively measurable processes $f(t, \omega, y, z)$ which are continuous in $(y, z)$ for almost all $(t, \omega)$ and measurable in $(t, \omega)$ for all $(y, z)$. We define an appropriate complete metric on it and then look at the prevalence, in the sense of Baire categories, of the set of all $f$ such that

(1) the corresponding BSDE $\left(E^{f, \xi}\right)$ has a unique solution;

(2) the approximate solutions, given by the successive approximations associated to $\left(E^{f, \xi}\right)$, converge to the unique solution of $\left(E^{f, \xi}\right)$;

(3) the solutions of equation $\left(E^{f, \xi}\right)$ (when they exist) are continuous with respect to the coefficient $f$.

It is shown, by using the Baire categories theorem, that the set of coefficients $f$ having the above three properties is a set of a second category of Baire. See Definition 2.2 below for the Baire category sets and Oxtoby's book [17] for more details on this subject. Since a set of the second category in a Baire space contains "almost all" the points of the space, it may be thought of as the topological analogue of the measure-theoretical concept of a set whose complement is of measure zero. Our results state that, in some sense, almost all BSDEs with bounded continuous coefficient have solutions which satisfy the 
above properties (1), (2), and (3). We do not impose any boundedness condition on the terminal data $\xi$ which is assumed to be square-integrable only.

The paper is organized as follows. Section 2 introduces some notations and definitions. Section 3 is devoted to the continuous dependence of solutions with respect to the coefficient. In Section 4, we deal with the continuous dependence of the solutions with respect to the coefficient. Section 5 is devoted to the convergence of the Picard successive approximations.

\section{Notations and definitions}

We denote by $\mathscr{E}$ the set of $\left(\mathbb{R}^{d} \times \mathbb{R}^{d \times r}\right)$-valued processes $(Y, Z)$ defined on $\mathbb{R}_{+} \times \Omega$, which are $\mathscr{F}_{t}$-adapted and such that

$$
\|(Y, Z)\|^{2}=E\left(\sup _{0 \leq t \leq 1}|Y(t)|^{2}+\int_{0}^{1}|Z(s)|^{2} d s\right)<+\infty .
$$

$(\mathscr{E},\|\cdot\|)$ is a Banach space.

Definition 2.1. A solution of equation $\left(E^{f, \xi}\right)$ is a pair $(Y, Z)$ which belongs to the space $(\mathscr{E},\|\cdot\|)$ and satisfies $\left(E^{f, \xi}\right)$.

Throughout the paper, the solutions of equation $\left(E^{f, \xi}\right)$ will be denoted by $\left(Y^{f}, Z^{f}\right)$. For a given real number $M>0$, we denote by $\mathscr{C}$ the set of functions $f(t, \omega, y, z)$, defined on $\mathbb{R}_{+} \times \Omega \times \mathbb{R}^{d} \times \mathbb{R}^{d \times r}$ with values in $\mathbb{R}^{d}$, which are continuous in $(y, z)$ for almost all $(t, \omega)$, measurable in $(t, \omega)$ for all $(y, z)$ and such that $\operatorname{esssup}_{(t, \omega, y, z)}|f(t, \omega, y, z)| \leq M$. Let $\mathscr{L}$ be the subset of $\mathscr{C}$ consisting of functions $f$ which are Lipschitz in $(y, z)$.

Definition 2.2. A Baire space is a separated topological space in which all countable intersections of dense open subsets are dense also. Let $T$ be a Baire space. A subset $F$ of $T$ is said to be meager (or a first-category set in the Baire sense) if it is contained in a countable union of closed nowhere dense subsets of $T$. The complement of a meager set is called a residual (or a second-category set).

\section{Prevalence of existence and uniqueness}

Since $f$ is bounded, we can assume without loss of generality that $\xi=0$. Indeed, let $(Y, Z)$ be a solution of the $\operatorname{BSDE}\left(E^{f, \xi}\right)$. By Itô's representation theorem, there exists a predictable process $Z^{\prime}$ such that $E\left(\xi / \mathscr{F}_{t}\right)=\xi+\int_{0}^{t} Z_{s}^{\prime} d W_{s}$. Thus, $Y_{t}-E\left(\xi / \mathscr{F}_{t}\right)=\int_{t}^{1} f\left(s, Y_{s}\right.$, $\left.Z_{s}\right) d s-\int_{t}^{1}\left(Z_{s}-Z_{s}^{\prime}\right) d W_{s}$. Define $Y_{t}^{\prime \prime}:=Y_{t}-E\left(\xi / \mathscr{F}_{t}\right), Z_{t}^{\prime \prime}:=Z_{t}-Z_{t}^{\prime}$, and $f^{\prime \prime}(s, u, v):=$ $f\left(s, u+E\left(\xi / \mathscr{F}_{s}\right), v+Z_{s}^{\prime}\right)$. It is not difficult to see that $(Y, Z)$ is a solution to the BSDE $\left(E^{(f, \xi)}\right)$ if and only if $\left(Y^{\prime \prime}, Z^{\prime \prime}\right)$ is a solution to the $\operatorname{BSDE}\left(E^{\left(f^{\prime \prime}, 0\right)}\right)$.

As a consequence, the terminal condition will play no role in our situation. Hence, we consider BSDEs with $\xi=0$.

We denote by $\mathscr{R}_{e}$ the set of processes $f \in \mathscr{C}$ for which equation $\left(E^{f, 0}\right)$ has a (not necessarily unique) solution and by $\mathscr{R}_{1}$ the subset of $\mathscr{C}$ which consists of all functions $f$ for which equation $\left(E^{f, 0}\right)$ has a unique solution. 
Theorem 3.1. $\mathscr{R}_{1}$ is a residual set in the Baire space $(\mathscr{b}, \rho)$.

To prove this theorem we need some lemmas.

LEMmA 3.2. Endowed with the distance

$$
\rho(f, g)=\sum_{n=1}^{\infty}\left(\frac{1}{2^{n}}\right) \frac{\left(E \int_{0}^{1} \sup _{|y|,|z| \leq n}|f(s, y, z)-g(s, y, z)|^{2} d s\right)^{1 / 2}}{1+\left(E \int_{0}^{1} \sup _{|y|,|z| \leq n}|f(s, y, z)-g(s, y, z)|^{2} d s\right)^{1 / 2}},
$$

$(\mathscr{C}, \rho)$ is a complete metric space in which $\mathscr{L}$ is dense.

The above lemma can be proved by truncation and regularization.

Lemma 3.3. Let $f$ be an element of $\mathscr{L}$ and let $\left(f_{n}\right)_{n \in \mathbb{N}}$ be a sequence in $\mathscr{R}_{e}$. Let $\left(\xi_{n}\right)_{n \in \mathbb{N}}$ be a sequence of square-integrable random variables which are $\mathscr{F}_{1}$-measurable. Assume that

$$
\rho\left(f_{n}, f\right) \longrightarrow 0, \quad E\left(\left|\xi_{n}-\xi\right|^{2}\right) \longrightarrow 0, \quad \text { as } n \longrightarrow \infty .
$$

Then $\left(Y^{f_{n}}, Z^{f_{n}}\right)$ converges to $\left(Y^{f}, Z^{f}\right)$ in $(\mathscr{E},\|\cdot\|)$.

Proof. Without loss of generality, we may suppose that $\xi=\xi_{n}=0$ for each $n$. Let $\left(Y^{f}, Z^{f}\right)$ (resp., $\left.\left(Y^{f_{n}}, Z^{f_{n}}\right)\right)$ be a solution of equation $\left(E^{f, 0}\right)$ (resp., $\left(E^{f_{n}, 0}\right)$ ). Itô's formula shows that

$$
\begin{aligned}
\mid Y_{t}^{f_{n}}- & \left.Y_{t}^{f}\right|^{2}+\int_{t}^{1}\left|Z_{s}^{f_{n}}-Z_{s}^{f}\right|^{2} d s \\
= & 2 \int_{t}^{1}\left(Y_{s}^{f_{n}}-Y_{s}^{f}\right)^{*}\left(f_{n}\left(s, Y_{s}^{f_{n}}, Z_{s}^{f_{n}}\right)-f\left(s, Y_{s}^{f}, Z_{s}^{f}\right)\right) d s \\
& \quad-\int_{t}^{1}\left(Y_{s}^{f_{n}}-Y_{s}^{f}\right)^{*}\left(Z_{s}^{f_{n}}-Z_{s}\right) d W_{s},
\end{aligned}
$$

where $\left(Y_{s}^{f_{n}}-Y_{s}^{f}\right) *$ denotes the transpose of the vector $\left(Y_{s}^{f_{n}}-Y_{s}^{f}\right)$. Let $\alpha$ be an arbitrary number in $\mathbb{R}_{+}^{*}$ and let $L$ be the Lipschitz constant of the function $f$. For a given positive number $N$, let $A_{n}^{N}=\left\{(s, \omega) ;\left|Y_{s}^{f_{n}}\right|^{2}+\left|Z_{s}^{f_{n}}\right|^{2}+\left|Y_{s}^{f}\right|^{2}+\left|Z_{s}^{f}\right|^{2} \geq N^{2}\right\}$ and $\bar{A}_{n}^{N}=\Omega \backslash A_{n}^{N}$, and denote by $\mathscr{X}_{E}$ the indicator function of the set $E$. Using Young and Chebychev inequalities and the fact that $f$ is uniformly Lipschitz, we get

$$
\begin{aligned}
& E\left(\left|Y_{t}^{f_{n}}-Y_{t}^{f}\right|^{2}\right)+E \int_{t}^{1}\left|Z_{s}^{f_{n}}-Z_{s}^{f}\right|^{2} d s \\
& \leq E \int_{t}^{1}\left|Y_{s}^{f_{n}}-Y_{s}^{f}\right|\left(\left|f_{n}\left(s, Y_{s}^{f_{n}}, Z_{s}^{f_{n}}\right)-f\left(s, Y_{s}^{f}, Z_{s}^{f}\right)\right|\right) d s \\
& \leq 2 \alpha^{2} E \int_{t}^{1}\left|Y_{s}^{f_{n}}-Y_{s}^{f}\right|^{2} d s \\
&+\frac{2}{\alpha^{2}} E \int_{t}^{1}\left|f_{n}\left(s, Y_{s}^{f_{n}}, Z_{s}^{f_{n}}\right)-f\left(s, Y_{s}^{f}, Z_{s}^{f}\right)\right|^{2} \mathscr{X}_{A_{n}^{N}} d s \\
&+\frac{2}{\alpha^{2}} E \int_{t}^{1}\left(\left|f_{n}\left(s, Y_{s}^{f_{n}}, Z_{s}^{f_{n}}\right)-f\left(s, Y_{s}^{f}, Z_{s}^{f}\right)\right|^{2}\right) \mathscr{X}_{\bar{A}_{n}^{N}(s)} d s
\end{aligned}
$$




$$
\begin{aligned}
\leq & 2 \alpha^{2} E \int_{t}^{1}\left|Y_{s}^{f_{n}}-Y_{s}^{f}\right|^{2} d s \\
& +\frac{8 M^{2}}{\alpha^{2}} \frac{1}{N^{2}} E \int_{t}^{1}\left(\left|Y_{s}^{f_{n}}\right|^{2}+\left|Z_{s}^{f_{n}}\right|^{2}+\left|Y_{s}^{f}\right|^{2}+\left|Z_{s}^{f}\right|^{2}\right) d s \\
& +\frac{4}{\alpha^{2}} E \int_{t}^{1}\left|f_{n}\left(s, Y_{s}^{f_{n}}, Z_{s}^{f_{n}}\right)-f\left(s, Y_{s}^{f_{n}}, Z_{s}^{f_{n}}\right)\right|^{2} \mathscr{X}_{\bar{A}_{n}^{N}(s)} d s \\
& +\frac{4}{\alpha^{2}} E \int_{t}^{1}\left|f\left(s, Y_{s}^{f_{n}}, Z_{s}^{f_{n}}\right)-f\left(s, Y_{s}^{f}, Z_{s}^{f}\right)\right|^{2} \mathscr{X}_{\bar{A}_{n}^{N}(s)} d s \\
\leq & 2 \alpha^{2} E \int_{t}^{1}\left|Y_{s}^{f_{n}}-Y_{s}^{f}\right|^{2} d s+\frac{8 M^{2} K}{\alpha^{2}} \frac{1}{N^{2}}+\frac{4}{\alpha^{2}}\left(\frac{2^{N} \rho\left(f_{n}, f\right)}{1-2^{N} \rho\left(f_{n}, f\right)}\right)^{2} d s \\
& +\frac{4}{\alpha^{2}} L^{2} E \int_{t}^{1}\left|Y_{s}^{f_{n}}-Y_{s}^{f}\right|^{2} d s+\frac{4}{\alpha^{2}} L^{2} E \int_{t}^{1}\left|Z_{s}^{f_{n}}-Z_{s}^{f}\right|^{2} d s,
\end{aligned}
$$

where $K$ is a constant which depends only on $M$.

We choose $\alpha$ such that $4 L^{2} / \alpha^{2}<1$, then we use Gronwall lemma to get $E\left(\mid Y^{f_{n}}(t)-\right.$ $\left.\left.Y^{f}(t)\right|^{2}\right) \leq\left[\left(4 / \alpha^{2}\right)\left(2^{N} \rho\left(f_{n}, f\right) / 1-2^{N} \rho\left(f_{n}, f\right)\right)^{2}+\left(8 M^{2} K / \alpha^{2}\right)\left(1 / N^{2}\right)\right] \exp \left(2 \alpha^{2}+1\right)$. We use Burkholder-Davis-Gundy inequality to show that a positive constant $C=C(\alpha, L)$ exists such that

$$
\begin{gathered}
E\left(\sup _{0 \leq t \leq 1}\left|Y^{f_{n}}(t)-Y^{f}(t)\right|^{2}\right) \leq C\left[\frac{4}{\alpha^{2}}\left(\frac{2^{N} \rho\left(f_{n}, f\right)}{1-2^{N} \rho\left(f_{n}, f\right)}\right)^{2}+\frac{8 M^{2} K}{\alpha^{2}} \frac{1}{N^{2}}\right] \exp \left(2 \alpha^{2}+1\right), \\
E \int_{0}^{1}\left|Z_{s}^{f_{n}}-Z_{s}^{f}\right|^{2} d s \leq C\left[\frac{4}{\alpha^{2}}\left(\frac{2^{N} \rho\left(f_{n}, f\right)}{1-2^{N} \rho\left(f_{n}, f\right)}\right)^{2}+\frac{8 M^{2} K}{\alpha^{2}} \frac{1}{N^{2}}\right] \exp \left(2 \alpha^{2}+1\right) .
\end{gathered}
$$

Lemma 3.3 follows by passing to the limit first on $n$ and next on $N$.

Now, we define the oscillation function $D_{\delta}: \mathscr{C} \rightarrow \mathbb{R}_{+}$as follows:

$$
\begin{gathered}
D_{\delta}(f)=\sup \left\{d\left(\left(Y^{f_{1}}, Z^{f_{1}}\right),\left(Y^{f_{2}}, Z^{f_{2}}\right)\right) ; f_{i} \in \mathscr{L} \text { and } \rho\left(f, f_{i}\right)<\delta \text { for } i=1,2\right\}, \\
D_{e}(f)=\lim _{\delta \rightarrow 0} D_{\delta}(f) .
\end{gathered}
$$

We then have the following lemma.

Lemma 3.4. (i) If $f$ belongs to $\mathscr{L}$, then $D_{e}(f)=0$.

(ii) The function $D_{e}$ is upper semicontinuous on $\mathscr{L}$.

Proof. Assertion (i) is a consequence of Lemma 3.3. We prove assertion (ii). Let $\left(f_{n}\right)$ be a sequence in $\mathscr{C}$ converging to a limit $f$, which belongs to $\mathscr{L}$. Assume that $\lim _{n \rightarrow \infty} D_{e}\left(f_{n}\right)>$ 0 . Then there exist $\epsilon>0$ and a subsequence $\left(n_{k}\right)$ such that, for each $k$, there exist two 
sequences $\left(f_{n_{k}}^{1}\right)$ and $\left(f_{n_{k}}^{2}\right)$ in $\mathscr{L}$, which satisfy

$$
\begin{gathered}
\rho\left(f_{n_{k}}, f_{n_{k}}^{1}\right)<\frac{1}{n_{k}}, \quad \rho\left(f_{n_{k}}, f_{n_{k}}^{2}\right)<\frac{1}{n_{k}}, \\
d\left(\left(Y^{f_{n_{k}}^{1}}, Z^{f_{n_{k}}^{1}}\right),\left(Y^{f_{n_{k}}^{2}}, Z^{f_{n_{k}}^{2}}\right)\right)>\epsilon .
\end{gathered}
$$

Thus, (3.7) and Lemma 3.3 imply that $\lim _{k \rightarrow \infty} d\left(\left(Y^{f_{n_{k}}^{1}}, Z^{f_{n_{k}}^{1}}\right),\left(Y^{f_{n_{k}}^{2}}, Z^{f_{n_{k}}^{2}}\right)\right)=0$. This contradicts (3.8). Assertion (ii) is proved.

The following proposition gives a sufficient condition which ensures the existence of solutions to the BSDE $\left(E^{f, 0}\right)$.

Proposition 3.5. If $D_{e}(f)=0$ for an $f$ in $\mathscr{C}$, then equation $\left(E^{f, 0}\right)$ has at least one solution in $\mathscr{E}$.

Proof. Let $f \in \mathscr{C}$. Since $D_{e}(f)=0$, then there exists a decreasing sequence of strictly positive numbers $\delta_{n}\left(\delta_{n} \downarrow 0\right)$ such that

$$
\sup \left\{d\left(\left(Y^{f_{1}}, Z^{f_{1}}\right),\left(Y^{f_{2}}, Z^{f_{2}}\right)\right) ; f_{i} \in \mathscr{L} \text { and } \rho\left(f, f_{i}\right)<\delta_{n} \text { for } i=1,2\right\}<\frac{1}{n} \text {. }
$$

But Lemma 3.2 implies that for each $n \in \mathbb{N}^{*}$, there exists $f_{n} \in \mathscr{L}$ such that $\rho\left(f_{n}, f\right)<$ $\delta_{n}$. Since $\delta_{n}$ decreases, it follows from (3.9) that $d\left(\left(Y^{f_{n}}, Z^{f_{n}}\right),\left(Y^{f_{m}}, Z^{f_{m}}\right)\right)<\sup (1 / m, 1 / n)$. Hence $\left(Y^{f_{n}}, Z^{f_{n}}\right)_{n \in \mathbb{N}}$ is a Cauchy sequence in the Banach space $(\mathscr{E},\|\cdot\|)$. Let $(Y, Z)$ be its limit. We will show that $(Y, Z)$ satisfies equation $\left(E^{f, 0}\right)$. We immediately have

$$
\begin{gathered}
\lim _{n \rightarrow \infty} E\left(\sup _{0 \leq s \leq 1}\left|Y^{f_{n}}(s)-Y(s)\right|^{2}\right)=0 \\
\lim _{n \rightarrow \infty} E \int_{0}^{1}\left|Z^{f_{n}}(s)-Z(s)\right|^{2} d s=0 .
\end{gathered}
$$

From (3.11) we get that, for each $t \in[0,1]$,

$$
\lim _{n \rightarrow \infty} \int_{t}^{1} Z^{f_{n}}(s) d W_{s}=\int_{t}^{1} Z(s) d W_{s} \quad \text { in probability. }
$$

Moreover, (3.10) and (3.11) imply that there exists a subsequence $\left(n_{k}\right)$ such that

$$
\left(Y^{f_{n_{k}}}, Z^{f_{n_{k}}}\right) \text { converges to }(Y, Z) \quad d P \times d t \text {-a.e. }
$$

It remains now to prove that, for each $t \in[0,1]$,

$$
\lim _{n \rightarrow \infty} \int_{t}^{1} f_{n_{k}}\left(s, Y^{f_{n_{k}}}(s), Z^{f_{n_{k}}}(s)\right) d s=\int_{t}^{1} f(s, Y(s), Z(s)) d s \quad \text { in probability. }
$$


Without loss of generality, we may assume that (3.13) holds without extracting subsequence. Let $N$ be an arbitrary positive number. Since both $f_{n}$ and $f$ are bounded by $M$, we can show that

$$
\begin{aligned}
E\left|\int_{t}^{1} f_{n}\left(s, Y^{f_{n}}(s), Z^{f_{n}}(s)\right) d s-\int_{t}^{1} f(s, Y(s), Z(s)) d s\right| \\
\leq E \int_{t}^{1}\left|f_{n}\left(s, Y^{f_{n}}(s), Z^{f_{n}}(s)\right)-f\left(s, Y^{f_{n}}(s), Z^{f_{n}}(s)\right)\right| d s \\
\quad+E \int_{0}^{1}\left|f\left(s, Y^{f_{n}}(s), Z^{f_{n}}(s)\right)-f(s, Y(s), Z(s))\right| d s \\
\leq E \int_{0}^{1} \sup _{|y|,|z| \leq N}\left|f_{n}(s, y, z)-f(s, y, z)\right| d s+\frac{2 M}{N} \\
\quad+E \int_{0}^{1}\left|f\left(s, Y^{f_{n}}(s), Z^{f_{n}}(s)\right)-f(s, Y(s), Z(s))\right| d s \\
=I_{1}(n)+\frac{2 M}{N}+I_{2}(n) .
\end{aligned}
$$

Lemma 3.2 shows that $\lim _{n \rightarrow \infty} I_{1}(n)=0$. On the other hand, since $f \in \mathscr{C}$, then (3.13) implies that $f\left(\cdot, Y^{f_{n}}(\cdot), Z^{f_{n}}(\cdot)\right)$ converges to $f(\cdot, Y(\cdot), Z(\cdot)), d P \times d s$-a.e. Hence, the Lebesgue dominated convergence theorem shows that $\lim _{n \rightarrow \infty} I_{2}(n)=0$. Proposition 3.5 is proved.

Proof of Theorem 3.1. Lemma 3.2 and assertions (i) and (ii) of Lemma 3.4 imply that, for each integer $n$, the set $\mathscr{G}_{n}=\left\{f \in \mathscr{b} ; D_{e}(f)<1 / n\right\}$ is a dense open subset of $(\mathscr{b}, \rho)$. Then, by the Baire categories theorem, the set $\mathscr{G}_{=}=\bigcap_{n \in \mathbb{N}^{*}} \mathscr{G}_{n}$ is a dense $G_{\delta}$ subset of the Baire space $(\mathscr{C}, \rho)$. Moreover, if $f \in \mathscr{G}$, then Proposition 3.5 implies that the corresponding equation $\left(E^{f, 0}\right)$ has one solution. Hence, $\mathscr{G}_{\subset} \subset \mathscr{R}_{e}$. This implies that $\mathscr{R}_{e}$ is a residual subset in $(\mathscr{C}, \rho)$.

To prove that $\mathscr{R}_{1}$ is residual, we define the function $D_{u}: \mathscr{G} \rightarrow \mathbb{R}_{+}$as follows: $D_{u}(f)=$ $\sup \left\{d\left(\left(Y_{1}^{f}, Z_{1}^{f}\right),\left(Y_{2}^{f}, Z_{2}^{f}\right)\right) ;\left(Y_{i}^{f}, Z_{i}^{f}\right)\right.$ is a solution to equation $\left.\left(E^{f, 0}\right), i=1,2\right\}$ and for each $n \in \mathbb{N}^{*}$, we put $\overline{\mathscr{G}}_{n}=\left\{f \in \mathscr{G}_{j} D_{u}(f)<1 / n\right\}$. By using Lemma 3.3, we see, as in the proof of Lemma 3.4(ii), that the function $D_{u}$ is upper semicontinuous on $\mathscr{L}$. This implies that each $\overline{\mathscr{G}}_{n}$ contains the intersection of $\mathscr{G}$ and a dense open subset of $(\mathscr{C}, \rho)$. Thus, the set $\overline{\mathscr{G}}=\bigcap_{n \in \mathbb{N}^{*}} \overline{\mathscr{G}}_{n}$ contains a dense $G_{\delta}$ subset of the Baire space $(\mathscr{C}, \rho)$. Hence, it is residual in $(\mathscr{C}, \rho)$. Finally, if $f \in \overline{\mathscr{G}}$, then the corresponding equation $\left(E^{f, 0}\right)$ has a unique solution. Thus, $\bar{G} \subset \mathscr{R}_{1}$. Theorem 3.1 follows.

Examples. In this section, we give two examples of BSDEs with nonuniformly Lipschitz coefficient and for which the existence and uniqueness hold. The proof follows as a direct application of Proposition 3.5. This shows that the sufficient condition given by the oscillation function $D_{e}(f)=0$ is not only theoretical but can also be applied to concrete cases. 
Corollary 3.6. Let $f \in \mathscr{C}$ and let $\xi$ be a square-integrable random variable. Assume moreover that $f$ satisfies the following hypothesis:

(H1) for every $N \in \mathbb{N}^{*}$, there exists a constant $L_{N}>0$ such that $\mid f(t, \omega, y, z)-f(t, \omega$, $\left.y^{\prime}, z^{\prime}\right) \mid \leq L_{N}\left(\left|y-y^{\prime}\right|+\left|z-z^{\prime}\right|\right), P$-a.s., a.e. $t \in[0,1]$, and for all $y, y^{\prime}, z$, $z^{\prime}$ such that $|y| \leq N,\left|y^{\prime}\right| \leq N,|z| \leq N,\left|z^{\prime}\right| \leq N$.

If $L_{N}=O(\sqrt{\log N})$, then equation $\left(E^{f, 0}\right)$ has a unique solution.

Proof. Let $\delta>0$ and $f_{1}, f_{2} \in \mathscr{L}$ be such that $\rho\left(f, f_{i}\right)<\delta, i=1,2$. Arguing as in the proof of Lemma 3.3, we show that there exists a constant $C=C(M, \xi)>0$ such that

$$
\begin{aligned}
& E\left(\sup _{0 \leq t \leq 1}\left|Y_{t}^{f_{1}}-Y_{t}^{f_{2}}\right|^{2}\right) \\
& \quad \leq C\left[\frac{2^{N} \rho^{2}\left(f_{1}, f\right)}{1-2^{N} \rho^{2}\left(f_{1}, f\right)}+\frac{2^{N} \rho^{2}\left(f_{2}, f\right)}{1-2^{N} \rho^{2}\left(f_{2}, f\right)}+\frac{1}{\left(L_{N}^{2}\right) N^{2}}\right] \exp \left(2 L_{N}^{2}\right)
\end{aligned}
$$

for each $N$ such that $N>1$ and $\rho\left(f, f_{i}\right)<1 / 2^{N}$.

Since $\rho\left(f_{i}, f\right)<\delta$ and $L_{N}=O(\sqrt{\log N})$, we deduce that

$$
D_{\delta}(f) \leq C\left[\frac{2^{N} \delta^{2}}{1-2^{N} \delta^{2}}+\frac{1}{L_{N}^{2}}\right]
$$

Letting $\delta \rightarrow 0$ and $N \rightarrow \infty$, we deduce that $D_{e}(f)=0$. Corollary 3.6 is proved.

Corollary 3.7. Let $f \in \mathscr{C}$ and let $\xi$ be a square-integrable random variable. Assume moreover that $f$ satisfies the following hypotheses:

(H2) for every $N \in \mathbb{N}$, there exists a constant $\mu_{N} \in \mathbb{R}$ such that $\left\langle y-y^{\prime}, f(t, \omega, y, z)-\right.$ $\left.f\left(t, \omega, y^{\prime}, z\right)\right\rangle \leq \mu_{N}\left|y-y^{\prime}\right|^{2}, P$-a.s., a.e. $t \in[0,1]$, and for all $y, y^{\prime}, z$ such that $|y| \leq$ $N,\left|y^{\prime}\right| \leq N,|z| \leq N$

(H3) for every $N \in \mathbb{N}$, there exists a constant $L_{N}>0$ such that $\left|f(t, \omega, y, z)-f\left(t, \omega, y, z^{\prime}\right)\right| \leq$ $L_{N}\left|z-z^{\prime}\right|, P$-a.s., a.e. $t \in[0,1]$, and for all $y, z, z^{\prime}$ such that $|y| \leq N,|z| \leq N$, $\left|z^{\prime}\right| \leq N$.

If $\mu_{N}^{+}+L_{N}^{2}=\mathcal{O}(\log N)$, then $\left(E^{(f, \xi)}\right)$ has a unique solution.

Proof. Let $\delta>0$ and $f_{1}, f_{2} \in \mathscr{L}$ be such that $\rho\left(f, f_{i}\right)<\delta, i=1,2$. Arguing as in the proof of Lemma 3.3, we show that there exists a constant $C=C(M, \xi)>0$ such that

$$
\begin{aligned}
& E\left(\sup _{0 \leq t \leq 1}\left|Y_{t}^{f_{1}}-Y_{t}^{f_{2}}\right|^{2}\right) \\
& \quad \leq C\left[\rho_{N}^{2}\left(f_{1}-f\right)+\rho_{N}^{2}\left(f_{2}-f\right)+\frac{1}{\left(2 \mu_{N}^{+}+L_{N}^{2}\right) N^{2}}\right] \exp \left(2 \mu_{N}^{+}+L_{N}^{2}\right)
\end{aligned}
$$

for each $N$ such that $N>1$ and $\rho\left(f, f_{i}\right)<1 / 2^{N}$. 
Since $\rho\left(f_{i}, f\right)<\delta$ and $\mu_{N}^{+}+L_{N}^{2}=\mathcal{O}(\log N)$, we deduce that

$$
D_{\delta}(f) \leq 2 C\left[\frac{2^{N} \delta^{2}}{1-2^{N} \delta^{2}}+\frac{1}{2 \mu_{N}^{+}+L_{N}^{2}}\right] .
$$

Letting $\delta \rightarrow 0$ and $N \rightarrow \infty$, we deduce that $D_{e}(f)=0$. Corollary 3.7 is proved.

\section{Continuous dependence on the coefficient}

For a given $f \in \mathscr{C}$, we denote by $S f=\left(Y^{f}, Z^{f}\right)$ the solution of $\left(E^{f, 0}\right)$ when it exists.

THeOREM 4.1. There exists a second-category set $\mathscr{R}_{2}$ such that the map $S: \mathscr{R}_{2} \rightarrow \mathscr{E}$, given by $S f=\left(Y^{f}, Z^{f}\right)$, is well defined and continuous at each point of $\mathscr{R}_{2}$.

Proof. We will show that $S$ is continuous on $\overline{\mathscr{G}}$ (the dense $G_{\delta}$ set which has been defined in the proof of Theorem 3.1). Suppose the contrary. Then there exist $f \in \bar{G}, \varepsilon>0$, and a sequence $\left(f_{p}\right) \subset \bar{G}$ such that

$$
\lim _{p \rightarrow \infty} \rho\left(f_{p}, f\right)=0, \quad d\left(S f_{p}, S f\right) \geq \varepsilon, \quad \text { for each } p .
$$

Fix $n \in \mathbb{N}$ such that $\varepsilon<1 / n$. Since $\overline{\mathscr{G}} \subset \mathscr{G}$, then there exist a decreasing sequence of strictly positive numbers $\delta_{n}\left(\delta_{n} \downarrow 0\right)$ and a sequence of functions $g_{n} \in \mathscr{L}$ such that

$$
\rho\left(g_{n}, f\right)<\delta_{n}, \quad d\left(S g_{n}, S f\right)<\frac{1}{n} .
$$

We choose $p$ large enough so as to have $\rho\left(f_{p}, f\right)<\delta_{n}-\rho\left(g_{n}, f\right)$, then we use (4.2) to obtain $\rho\left(f_{p}, g_{n}\right)<\delta_{n}$. Therefore, $d\left(S f_{p}, S g_{n}\right)<1 / n$. Thus, $d\left(S f_{p}, S f\right) \leq d\left(S f_{p}, S g_{n}\right)+d\left(S g_{n}, S f\right)$ $<1 / n+1 / n<(2 / 3) \varepsilon$, which contradicts (4.1). Theorem 4.1 is proved.

\section{The Picard successive approximations}

For a given $f \in \mathscr{C}$, we denote by $\left(Y_{n}^{f}, Z_{n}^{f}\right)$ the sequence of processes defined by the following equation:

$\left(E_{n}^{f}\right)$

$$
Y_{0}^{f}(t)=Z_{0}^{f}(t)=0, \quad Y_{n+1}^{f}(t)=\int_{t}^{1} f\left(s, Y_{n}^{f}(s), Z_{n}^{f}(s)\right) d s-\int_{t}^{1} Z_{n+1}^{f}(s) d W_{s} .
$$

Itô's representation theorem shows that the sequence $\left(Y_{n}^{f}, Z_{n}^{f}\right)$ is well defined for each $n$. Let $\mathscr{R}_{3}$ be the subset of $\mathscr{C}$ of all those $f \in \mathscr{C}$ such that the corresponding sequence $\left(Y_{n}^{f}, Z_{n}^{f}\right)$, defined by $\left(E_{n}^{f, 0}\right)$, converges in $(\mathscr{E},\|\cdot\|)$ to a solution $\left(Y^{f}, Z^{f}\right)$ of equation $\left(E^{f, 0}\right)$.

Theorem 5.1. The set $\mathscr{R}_{3}$ is residual in $(\mathscr{b}, \rho)$.

To prove this theorem, we need the following lemma which is the analogue of the previous Lemma 3.3. 
Lemma 5.2. Let $f$ be an element of $\mathscr{L}$ and let $\left(f_{p}\right)_{p \in \mathbb{N}}$ be a sequence in $\mathscr{R}_{3}$. Denote by $\left(Y_{n}^{f}, Z_{n}^{f}\right)$ (resp., $\left(Y_{n}^{f_{p}}, Z_{n}^{f_{p}}\right)$ ) the sequence defined by equation $\left(E_{n}^{f}\right)$ (resp., $\left(E_{n}^{f_{p}}\right)$ ). Assume that $\rho\left(f_{p}, f\right) \rightarrow 0$ as $p \rightarrow \infty$. Then $\lim _{p \rightarrow \infty} \sup _{n \in \mathbb{N}}\left\|\left(Y_{n}^{f_{p}}, Z_{n}^{f_{p}}\right)-\left(Y_{n}^{f}, Z_{n}^{f}\right)\right\|=0$.

Proof. Let $\left(Y_{n}^{f}, Z_{n}^{f}\right)$ (resp., $\left.\left(Y_{n}^{f_{p}}, Z_{n}^{f_{p}}\right)\right)$ be a solution of equation $\left(E_{n}^{f}\right)$ (resp., $\left.\left(E_{n}^{f_{p}}\right)\right)$. Itô's formula shows that

$$
\begin{aligned}
& E\left(\left|Y_{n+1}^{f_{p}}(t)-Y_{n+1}^{f}(t)\right|^{2}\right)+E \int_{t}^{1}\left|Z_{n+1}^{f_{p}}(s)-Z_{n+1}^{f}(s)\right|^{2} d s \\
& \quad=2 E \int_{t}^{1}\left(Y_{n+1}^{f_{p}}(s)-Y_{n+1}^{f}(s)\right)^{*}\left(f_{p}\left(s, Y_{n}^{f_{p}}(s), Z_{n}^{f_{p}}(s)\right)-f\left(s, Y_{n}^{f}(s), Z_{n}^{f}(s)\right)\right) d s
\end{aligned}
$$

where $\left(Y_{n+1}^{f_{p}}(s)-Y_{n+1}^{f}(s)\right)^{*}$ denotes the transpose of the vector $\left(Y_{n+1}^{f_{p}}(s)-Y_{n+1}^{f}(s)\right)$. Let $\alpha$ be an arbitrary number in $\mathbb{R}_{+}^{*}$ and let $L$ be the Lipschitz constant of the function $f$. For a given positive number $N$, let $A_{n, p}^{N}=\left\{(s, \omega) ;\left|Y_{n}^{f_{p}}(s)\right|^{2}+\left|Z_{n}^{f_{p}}(s)\right|^{2}+\left|Y_{n}^{f}(s)\right|^{2}+\left|Z_{n}^{f}(s)\right|^{2} \geq\right.$ $\left.N^{2}\right\}$ and $\bar{A}_{n, p}^{N}=\Omega \backslash A_{n, p}^{N}$, and denote by $\mathscr{X}_{E}$ the indicator function of the set $E$. Arguing as in the proof of Lemma 3.3, we obtain the following inequalities:

$$
\begin{aligned}
& E\left(\left|Y_{n+1}^{f_{p}}(t)-Y_{n+1}^{f}(t)\right|^{2}\right)+E \int_{t}^{1}\left|Z_{n+1}^{f_{p}}(s)-Z_{n+1}^{f}(s)\right|^{2} d s \\
& \leq 2 \alpha^{2} E \int_{t}^{1}\left|Y_{n+1}^{f_{p}}(s)-Y_{n+1}^{f}(s)\right|^{2} d s \\
&+\frac{2}{\alpha^{2}} E \int_{t}^{1}\left|f_{p}\left(s, Y_{n}^{f_{p}}(s), Z_{n}^{f_{p}}(s)\right)-f\left(s, Y_{n}^{f}(s), Z_{n}^{f}(s)\right)\right|^{2} \mathscr{X}_{A_{n, p}^{N}} d s \\
&+\frac{2}{\alpha^{2}} E \int_{t}^{1}\left(\left|f_{p}\left(s, Y_{n}^{f_{p}}(s), Z_{n}^{f_{p}}(s)\right)-f\left(s, Y_{n}^{f}(s), Z_{n}^{f}(s)\right)\right|^{2}\right) \mathscr{X}_{\bar{A}_{n, p}^{N}} d s \\
& \leq 2 \alpha^{2} E \int_{t}^{1}\left|Y_{n+1}^{f_{p}}(s)-Y_{n+1}^{f}(s)\right|^{2} d s \\
&+\frac{8 M^{2}}{\alpha^{2}} \frac{1}{N^{2}} E \int_{t}^{1}\left(\left|Y_{n}^{f_{p}}(s)\right|^{2}+\left|Z_{n}^{f_{p}}(s)\right|^{2}+\left|Y_{n}^{f}(s)\right|^{2}+\left|Z_{n}^{f}(s)\right|^{2}\right) d s \\
&+\frac{4}{\alpha^{2}} E \int_{t}^{1}\left|f_{p}\left(s, Y_{n}^{f_{p}}(s), Z_{n}^{f_{p}}(s)\right)-f\left(s, Y_{n}^{f_{p}}(s), Z_{n}^{f_{p}}(s)\right)\right|^{2} \mathscr{X}_{\bar{A}_{n, p}^{N}} d s \\
&+\frac{4}{\alpha^{2}} E \int_{t}^{1}\left|f\left(s, Y_{n}^{f_{p}}(s), Z_{n}^{f_{p}}(s)\right)-f\left(s, Y_{n}^{f}(s), Z_{n}^{f}(s)\right)\right|^{2} \mathscr{X}_{\bar{A}_{n, p}^{N}} d s \\
& \leq 2 \alpha^{2} E \int_{t}^{1}\left|Y_{n+1}^{f_{p}}(s)-Y_{n+1}^{f}(s)\right|^{2} d s \\
&+\frac{8 M^{2} K}{\alpha^{2}} \frac{1}{N^{2}}+\frac{4}{\alpha^{2}}\left(\frac{2^{N} \rho\left(f_{p}, f\right)}{1-2^{N} \rho\left(f_{p}, f\right)}\right)^{2} d s \\
&+\frac{4}{\alpha^{2}} L^{2} E \int_{t}^{1}\left|Y_{n}^{f_{p}}(s)-Y_{n}^{f}(s)\right|^{2} d s+\frac{4}{\alpha^{2}} L^{2} E \int_{t}^{1}\left|Z_{n}^{f_{p}}(s)-Z_{n}^{f}(s)\right|^{2} d s
\end{aligned}
$$

where $K$ is a constant which depends only on $M$. 
We put $\varphi_{n}^{p}(t)=\sup _{t \leq u \leq 1} E\left(\left|Y_{n}^{f_{p}}(u)-Y_{n}^{f}(u)\right|^{2}\right)+E \int_{t}^{1}\left|Z_{n}^{f_{p}}(s)-Z_{n}^{f}(s)\right|^{2} d s$, then we have

$$
\varphi_{n+1}^{p}(t) \leq 2 \alpha^{2} \int_{t}^{1} \varphi_{n+1}^{p}(s) d s+\frac{4 L^{2}}{\alpha^{2}} \varphi_{n}^{p}(t)+\frac{8 M^{2} K}{\alpha^{2}} \frac{1}{N^{2}}+\frac{4}{\alpha^{2}}\left(\frac{2^{N} \rho\left(f_{p}, f\right)}{1-2^{N} \rho\left(f_{p}, f\right)}\right)^{2}
$$

and Gronwall lemma implies that

$$
\varphi_{n+1}^{p}(t) \leq \frac{4 L^{2}}{\alpha^{2}} \varphi_{n}^{p}(t) \exp 2 \alpha^{2}(1-t)+\left[\frac{8 M^{2} K}{\alpha^{2}} \frac{1}{N^{2}}+\frac{4}{\alpha^{2}}\left(\frac{2^{N} \rho\left(f_{p}, f\right)}{1-2^{N} \rho\left(f_{p}, f\right)}\right)^{2}\right] \exp 2 \alpha^{2}
$$

If we choose $\alpha^{2}=12 L^{2}$ and $t$ sufficiently close to 1 so as to have $\exp \left(24 L^{2}(1-t)\right) \leq 3 / 2$, we obtain

$$
\varphi_{n+1}^{p}(t) \leq \frac{1}{2} \varphi_{n}^{p}(t)+\left[\frac{8 M^{2} K}{12 L^{2}} \frac{1}{N^{2}}+\frac{4}{12 L^{2}}\left(\frac{2^{N} \rho\left(f_{p}, f\right)}{1-2^{N} \rho\left(f_{p}, f\right)}\right)^{2}\right] \exp \left(24 L^{2}\right) .
$$

Since $\varphi_{0}^{p}(t)=0$ for each $t$ and $p$, we deduce that

$$
\sup _{n \in \mathbb{N}} \varphi_{n}^{p}(t) \leq\left[\frac{8 M^{2} K}{12 L^{2}} \frac{1}{N^{2}}+\frac{4}{12 L^{2}}\left(\frac{2^{N} \rho\left(f_{p}, f\right)}{1-2^{N} \rho\left(f_{p}, f\right)}\right)^{2}\right] \exp \left(24 L^{2}\right) .
$$

We successively pass to the limit in $p$ and $N$ to get $\lim _{p \rightarrow \infty} \sup _{n \in \mathbb{N}} \varphi_{n}^{p}(t)=0$ for each $t$ such that $\exp \left(24 L^{2}(1-t)\right) \leq 3 / 2$. Iterating this procedure on the subintervals $\left[t_{i}, t_{i+1}\right]$ such that $\exp \left(24 L^{2}\left(t_{i+1}-t_{i}\right)\right) \leq 3 / 2$, we obtain $\lim _{p \rightarrow \infty} \sup _{n \in \mathbb{N}} \varphi_{n}^{p}(t)=0$ for each $t \in[0,1]$. To finish the proof, we use Burkholder-Davis-Gundy inequality. Lemma 5.2 is proved.

Proof of Theorem 5.1. Let $f^{\prime} \in \mathscr{L}$ and $k \in \mathbb{N}^{*}$. By Lemma 5.2, there exists $\delta\left(f^{\prime}, k\right)>0$ such that, for every $f \in \mathscr{C}$ satisfying $\rho\left(f^{\prime}, f\right)<\delta\left(f^{\prime}, k\right)$, the inequality $\|\left(Y_{n}^{f^{\prime}}, Z_{n}^{f^{\prime}}\right)-$ $\left(Y_{n}^{f}, Z_{n}^{f}\right) \|<1 / k$ holds. By Lemma 3.2 and the Baire categories theorem, the set $\mathscr{G}_{1}=$ $\bigcap_{k} \cup_{f^{\prime} \in \mathscr{L}}\left\{f \in \mathscr{C} ; \rho\left(f^{\prime}, f\right)<\delta\left(f^{\prime}, k\right)\right\}$ is a dense $G_{\delta}$ subset in the Baire space $(\mathscr{C}, \rho)$. We will prove that for each $f \in \mathscr{G}_{1}$, the sequence $\left(Y_{n}^{f}, Z_{n}^{f}\right)$ defined by $\left(E_{n}^{f}\right)$ converges, in $(\mathscr{E},\|\cdot\|)$, to a solution of equation $\left(E^{f, 0}\right)$. Let $f \in \mathscr{G}_{1}$ and $\varepsilon>0$. We use Lemma 5.2 and the fact that the sequence $\left(Y_{n}^{f^{\prime}}, Z_{n}^{f^{\prime}}\right)$ converges for $f^{\prime} \in \mathscr{L}$ to show that a positive number $N_{0}$ exists such that, for any $n, m \geq N_{0}$, the following inequality holds:

$$
\begin{aligned}
\left\|\left(Y_{n}^{f}, Z_{n}^{f}\right)-\left(Y_{m}^{f}, Z_{m}^{f}\right)\right\| \leq & \left\|\left(Y_{n}^{f}, Z_{n}^{f}\right)-\left(Y_{n}^{f^{\prime}}, Z_{n}^{f^{\prime}}\right)\right\|+\left\|\left(Y_{n}^{f^{\prime}}, Z_{n}^{f^{\prime}}\right)-\left(Y_{m}^{f^{\prime}}, Z_{m}^{f^{\prime}}\right)\right\| \\
& +\left\|\left(Y_{m}^{f^{\prime}}, Z_{m}^{f^{\prime}}\right)-\left(Y_{m}^{f}, Z_{m}^{f}\right)\right\|<3 \varepsilon .
\end{aligned}
$$

Hence, $\left(Y_{n}^{f}, Z_{n}^{f}\right)$ is a Cauchy sequence in the Banach space $(\mathscr{E},\|\cdot\|)$, and so its convergence follows. Let $(Y, Z)$ be its limit. We will show that $(Y, Z)$ satisfies equation $\left(E^{f, 0}\right)$. 
Since $\left(Y_{n}^{f}, Z_{n}^{f}\right)$ converges to $(Y, Z)$ in the space $(\mathscr{E},\|\cdot\|)$, we immediately have

$$
\lim _{n \rightarrow \infty} E\left(\sup _{0 \leq t \leq 1}\left|Y_{n+1}^{f}(t)-Y(t)\right|^{2}\right)=0, \quad \lim _{n \rightarrow \infty} E \int_{0}^{1}\left|Z_{n+1}^{f}(s)-Z(s)\right|^{2} d s=0 .
$$

We prove that $\lim _{n \rightarrow \infty} E \int_{0}^{1}\left|f\left(s, Y_{n}^{f}(s), Z_{n}^{f}(s)\right)-f(s, Y(s), Z(s))\right|^{2} d s=0$. Since $f$ is bounded by $M$, we have $E\left(\left|Y_{n}^{f}(t)\right|^{2}\right) \leq M^{2}$, and then by Fatou's lemma, we obtain $E\left(\mid Y_{n}^{f}(t)-\right.$ $\left.\left.Y(t)\right|^{2}\right) \leq 2 M^{2}$. Hence, by (5.9), the sequence $\left(Y_{n}^{f}, Z_{n}^{f}\right)$ converges to $(Y, Z)$ in $L^{2}([0,1] \times$ $\Omega)$. Since $f$ is bounded and continuous, then $\lim _{n \rightarrow \infty} E \int_{o}^{1} \mid f\left(s, Y_{n}^{f}(s), Z_{n}^{f}(s)\right)-f(s, Y(s)$, $Z(s))\left.\right|^{2} d s=0$. Theorem 5.1 is proved.

Remark 5.3. The prevalence of the continuity of the solution with respect to the coefficient can also be proved via the Picard successive approximations.

Indeed, let $\overline{\mathscr{G}}$ and $\mathscr{G}_{1}$ be the residual sets defined in the proofs of Theorems 3.1 and 5.1, respectively. Put $\mathscr{G}_{2}:=\overline{\mathscr{G}} \cap \mathscr{G}_{1}$ and let $S: \mathscr{R}_{3} \mapsto \mathscr{E}$ be given by $S f=\left(Y^{f}, Z^{f}\right)$. We will prove that $S$ is continuous in $\mathscr{G}_{2}$, the residual set defined in the proof of Theorem 5.1. Assume the contrary holds. Then there exist $f \in \mathscr{G}_{2}, \varepsilon>0$, and a sequence $\left(f_{p}\right) \subset \mathscr{G}_{2}$ such that

$$
\lim _{p \rightarrow \infty} \rho\left(f_{p}, f\right)=0, \quad\left\|S f_{p}-S f\right\| \geq \varepsilon, \quad \text { for each } p .
$$

Let $k \in \mathbb{N}^{*}$ such that $1 / k<\mathcal{E} / 4$. Since $f \in \mathscr{G}_{2} \subset \mathscr{G}_{1}$, there exists a sequence $\left(g_{k}\right) \subset \mathscr{L}$ such that

$$
\rho\left(g_{k}, f\right)<\delta\left(g_{k}, k\right)
$$

Hence, by Lemma 5.2, we have $\left\|\left(Y_{n}^{g_{k}}, Z_{n}^{g_{k}}\right)-\left(Y_{n}^{f}, Z_{n}^{f}\right)\right\|<1 / k$ for each $n \in \mathbb{N}^{*}$. Passing to the limit on $n$, we show by using Theorem 5.1 that

$$
\left\|S g_{k}-S f\right\| \leq \frac{1}{k}
$$

We choose $p$ large enough such that $\rho\left(f_{p}, f\right)<\delta\left(g_{k}, k\right)-\rho\left(g_{k}, f\right)$, then we use (3.8) and the triangular inequality to get $\rho\left(f_{p}, g_{k}\right) \leq \delta\left(g_{k}, k\right)$. Hence, by using Lemma 3.3, we obtain $\left\|\left(Y_{n}^{g_{k}}, Z_{n}^{g_{k}}\right)-\left(Y_{n}^{f_{p}}, Z_{n}^{f_{p}}\right)\right\|<1 / k$ for each $n \in \mathbb{N}^{*}$. We pass to the limit on $n$ and use Theorem 5.1 to get $\left\|S g_{k}-S f_{p}\right\| \leq 1 / k$. Thus, $\left\|S f_{p}-S f\right\| \leq\left\|S f_{p}-S g_{k}\right\|+\left\|S g_{k}-S f\right\| \leq$ $2 / k<\varepsilon / 2$, which contradicts (3.7).

\section{Acknowledgments}

The first author was partially supported by CMEP 077/2001, AI no. MA/01/02. The second author was partially supported by CNRS/DEF, PICS 444, and MENA SwedishAlgerian Research Partnership Program (348-2002-6874). The third author was partially supported by CMIFM, AI no. MA/01/02. 


\section{References}

[1] J. J. Alibert and K. Bahlali, Genericity in deterministic and stochastic differential equations, Séminaire de Probabilités, XXXV, Lecture Notes in Math., vol. 1755, Springer, Berlin, 2001, pp. 220-240.

[2] K. Bahlali, Existence and uniqueness of solutions for BSDEs with locally Lipschitz coefficient, Electron. Comm. Probab. 7 (2002), 169-179.

[3] K. Bahlali, E. H. Essaky, M. Hassani, and E. Pardoux, Existence, uniqueness and stability of backward stochastic differential equations with locally monotone coefficient, C. R. Math. Acad. Sci. Paris 335 (2002), no. 9, 757-762.

[4] K. Bahlali, B. Mezerdi, and Y. Ouknine, Some generic properties of stochastic differential equations, Stochastics Stochastics Rep. 57 (1996), no. 3-4, 235-245.

[5] J.-M. Bismut, Conjugate convex functions in optimal stochastic control, J. Math. Anal. Appl. 44 (1973), 384-404.

[6] F. S. De Blasi and J. Myjak, Generic properties of differential equations in a Banach space, Bull. Acad. Polon. Sci. Sér. Sci. Math. Astronom. Phys. 26 (1978), no. 5, 395-400.

[7] A. Dermoune, S. Hamadène, and Y. Ouknine, Backward stochastic differential equation with local time, Stochastics Stochastics Rep. 66 (1999), no. 1-2, 103-119.

[8] N. El Karoui, S. Peng, and M. C. Quenez, Backward stochastic differential equations in finance, Math. Finance 7 (1997), no. 1, 1-71.

[9] P. R. Halmos, In general a measure preserving transformation is mixing, Ann. of Math. (2) 45 (1944), 786-792.

[10] S. Hamadène, Équations différentielles stochastiques rétrogrades: les cas localement lipschitzien [Backward stochastic differential equations: the locally Lipschitz case], Ann. Inst. H. Poincaré Probab. Statist. 32 (1996), no. 5, 645-659 (French).

[11] A. J. Heunis, On the prevalence of stochastic differential equations with unique strong solutions, Ann. Probab. 14 (1986), no. 2, 653-662.

[12] M. Kobylanski, Résultats d'existence et d'unicité pour des équations différentielles stochastiques rétrogrades avec des générateurs à croissance quadratique [Existence and uniqueness results for backward stochastic differential equations when the generator has quadratic growth], C. R. Acad. Sci. Paris Sér. I Math. 324 (1997), no. 1, 81-86 (French).

[13] A. Lasota and J. A. Yorke, The generic property of existence of solutions of differential equations in Banach space, J. Differential Equations 13 (1973), 1-12.

[14] J. P. Lepeltier and J. San Martin, Backward stochastic differential equations with continuous coefficient, Statist. Probab. Lett. 32 (1997), no. 4, 425-430.

[15] X. Mao, Adapted solutions of backward stochastic differential equations with non-Lipschitz coeffcients, Stochastic Process. Appl. 58 (1995), no. 2, 281-292.

[16] W. Orlicz, Zur Theorie der Differentialgleichung $y^{\prime}=f(x, y)$, Bull. Int. Acad. Polon. Sci. A (1932), no. 8-9, 221-228 (German).

[17] J. C. Oxtoby, Measure and Category. A Survey of the Analogies between Topological and Measure Spaces, Graduate Texts in Mathematics, vol. 2, Springer-Verlag, New York, 1971.

[18] É. Pardoux and S. Peng, Adapted solution of a backward stochastic differential equation, Systems Control Lett. 14 (1990), no. 1, 55-61.

[19]__ Backward stochastic differential equations and quasilinear parabolic partial differential equations, Stochastic Partial Differential Equations and Their Applications (Charlotte, NC, 1991) (B. L. Rozovskiı̌ and R. B. Sowers, eds.), Lecture Notes in Control and Inform. Sci., vol. 176, Springer, Berlin, 1992, pp. 200-217.

[20] S. Peng, Probabilistic interpretation for systems of quasilinear parabolic partial differential equations, Stochastics Stochastics Rep. 37 (1991), no. 1-2, 61-74.

[21] V. Rohlin, A "general" measure-preserving transformation is not mixing, Doklady Akad. Nauk SSSR (N.S.) 60 (1948), 349-351 (Russian). 


\section{Prevalence of BSDE}

[22] B. Simon, Operators with singular continuous spectrum. I. General operators, Ann. of Math. (2) 141 (1995), no. 1, 131-145.

[23] A. V. Skorokhod, Stochastic differential equations depending on a parameter, Theory Probab. Appl. 25 (1981), 659-666.

K. Bahlali: UFR Sciences, Université de Toulon et du Var, BP 132, 83957 La Garde Cedex, France Current address: Centre de Physique Théorique, Centre National de la Recherche Scientifique Luminy, Case 907, 13288 Marseille Cedex 9, France

E-mail address: bahlali@univ-tln.fr

B. Mezerdi: Département de Mathématiques, Université Mohamed Khider, BP 145, 07000 Biskra, Algeria

E-mail address: bmezerdi@wissal.dz

Y. Ouknine: Département de Mathématiques, Faculté des Sciences Semlalia, Université Cadi Ayyad, BP 2390, 40000 Marrakesh, Morocco

E-mail address: ouknine@ucam.ac.ma 


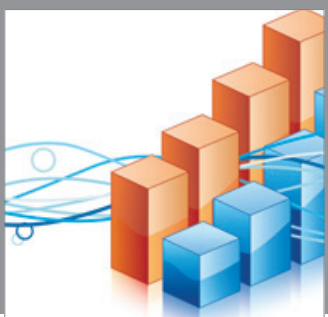

Advances in

Operations Research

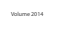

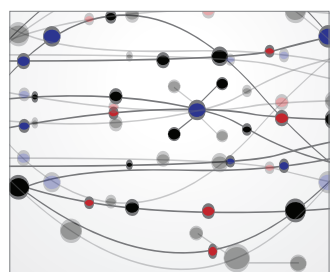

\section{The Scientific} World Journal
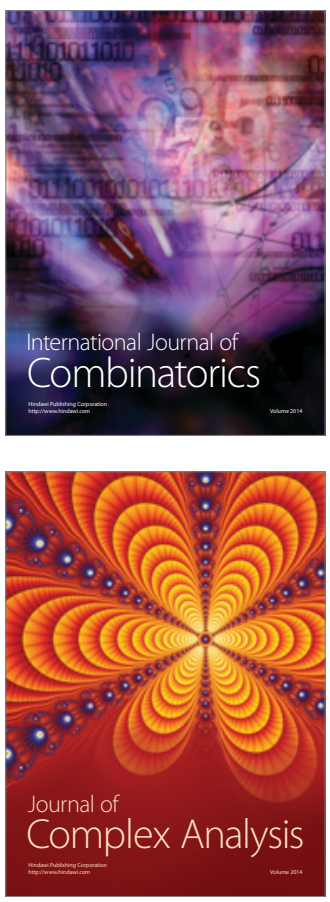

International Journal of

Mathematics and

Mathematical

Sciences
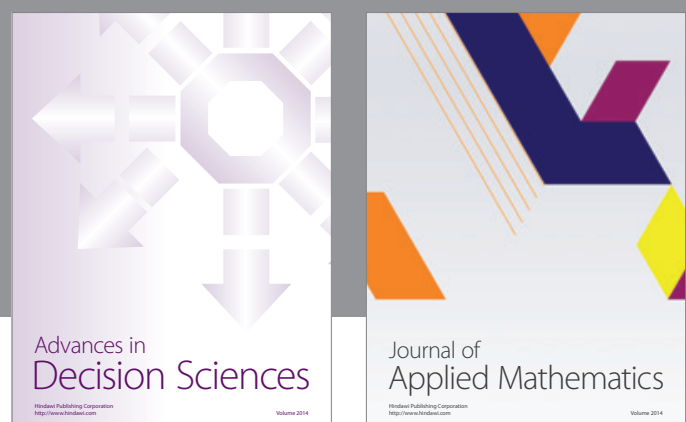

Journal of

Applied Mathematics
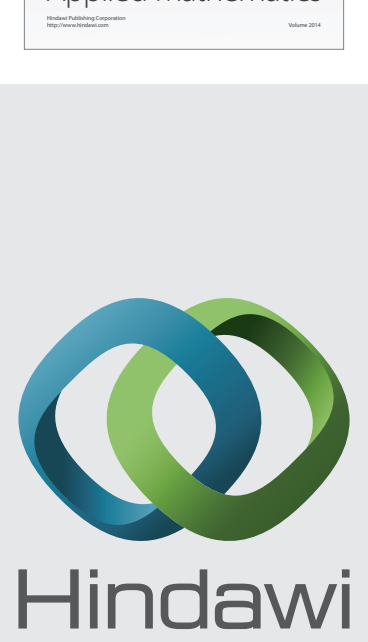

Submit your manuscripts at http://www.hindawi.com
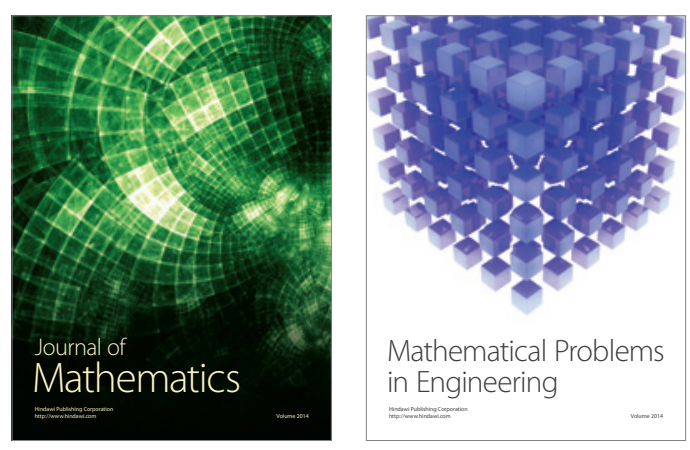

Mathematical Problems in Engineering
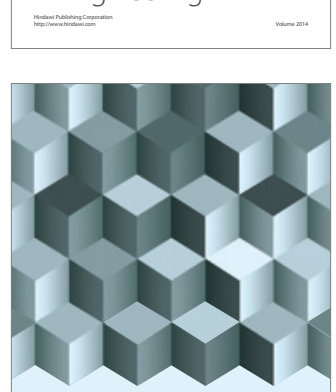

Journal of

Function Spaces
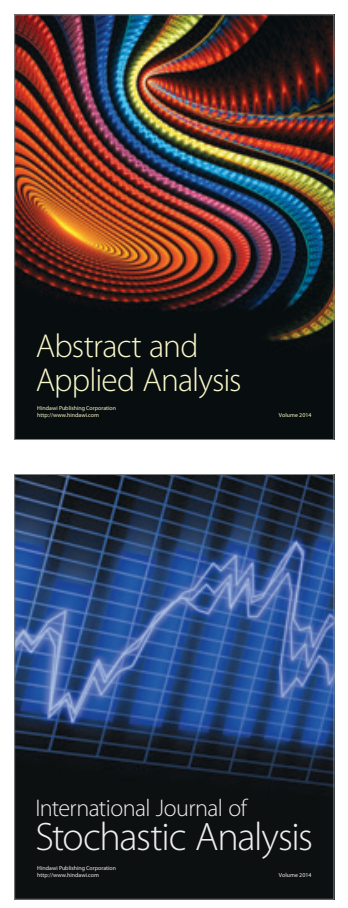

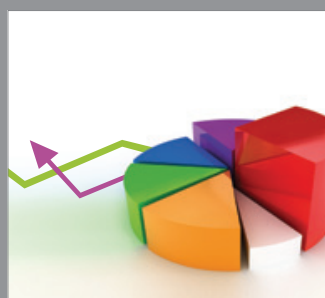

ournal of

Probability and Statistics

Promensencen
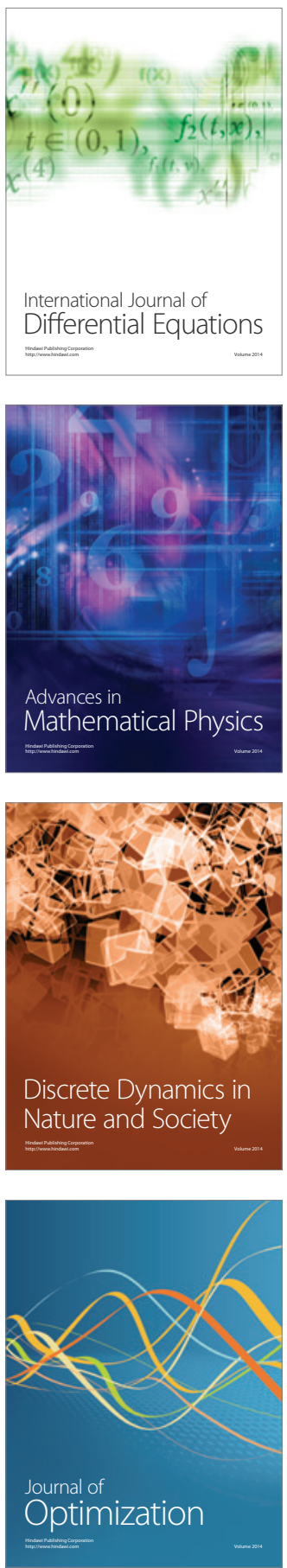